\section{JTI}

JOURNAL OF

TRAUMA AND INJURY

\title{
Priority Setting in Damage Control Surgery for Multiple Abdominal Trau- ma Following Resuscitative Endovas- cular Balloon Occlusion of the Aorta
}

\author{
Yoonjung Heo, M.D., Seok Won Lee, M.D., Dong Hun Kim, M.D. \\ Department of Trauma Surgery, Trauma Center, Dankook University Hospital, Cheonan, \\ Korea
}

Received: August 14, 2020

Revised: September 8, 2020

\section{Correspondence to}

Dong Hun Kim, M.D.

Department of Trauma Surgery, Trauma Center, Dankook University Hospital, 201 Manghyang-ro, Dongnam-gu, Cheonan 31116 , Korea

Tel: $+82-41-550-7119$

Fax: +82-41-550-0039

E-mail: saint7331@gmail.com
Damage control surgery (DCS) is an abbreviated laparotomy procedure that focuses on controlling bleeding to limit the surgical insult. It has become the primary treatment modality for patients with exsanguinating truncal trauma. Herein, we present the case of a 47-year-old woman with liver, kidney, and superior mesenteric vein (SMV) injuries caused by a motor vehicle collision. The patient underwent DCS following resuscitative endovascular balloon occlusion of the aorta (REBOA). In this case report, we discuss the importance of priority setting in DCS for the treatment of multisystem damage of several abdominal organs, particularly when the patient has incurred a combination of major vascular injuries. We also discuss the implications of damage control of the SMV, perihepatic packing, and right-sided medial visceral rotation. Further understanding of DCS, along with REBOA as a novel resuscitation strategy, can facilitate the conversion of uniformly lethal abdominal injuries into rescuable injuries.

Keywords: Mesenteric veins; Liver; Kidney; Balloon occlusion; Surgical procedures, operative

\section{INTRODUCTION}

Perihepatic packing with planned reoperation was described as the first technique of damage control surgery (DCS) in the early 1900s [1]. Ever since, DCS has been performed as a standard of care. It comprises an abbreviated operation, intensive care unit resuscitation, and then return to the operating room for a definitive surgery to prevent the lethal triad of death (hypothermia, acidosis, and coagulopathy) in patients (http://creativecommons.org/licenses/by-nc/4.0/) which permits unrestricted noncommercial use, distribution, and reproduction in any medium, provided the original work is properly cited. 


\section{JTI}

with trauma [2]. Controlling catastrophic bleeding should be the top priority in DCS; however, a goal-directed strategy of setting up priorities is essential when multiple organs are severely injured at the same time. Resuscitative endovascular balloon occlusion of the aorta (REBOA) is a novel resuscitative procedure that can be performed prior to DCS to decrease distal arterial flow to injured organs or vessels below the diaphragm. An increasing number of patients with exsanguinating torso trauma get the opportunity to be brought to the operating room with an inflated REBOA [3]. Herein, we present the case of a hemodynamically unstable patient with combined liver, kidney, and superior mesenteric vein (SMV) injuries who was stabilized with REBOA in Zone I of the aorta. SMV injury is rare, accounting for approximately $0.1 \%$ in patients with multiple traumas. Nevertheless, this situation requires a more concise and effective treatment strategy because the injury results in a high mortality rate [4-6]. The anatomic proximity of the SMV to other numerous vessels and organs accounts for the high incidence of concomitant injuries [5], thereby presenting a perplexing challenge to the surgeon performing DCS. As an optimal guideline for the treatment of SMV injury has not yet been established, the purpose of this report is to review the order of priority in DCS for patients with multiple traumatic abdominal damages, including superior mesenteric vessel injuries.

\section{CASE REPORT}

The patient was a 47-year-old woman who was an unre- strained driver hitting a guardrail. She was transferred to our regional level I trauma center 130 minutes after the accident. The patient initially presented as hemodynamically compromised with a blood pressure of $78 / 40 \mathrm{mmHg}$ and a heart rate of 110 beats per minutes. Distension and tenderness over the whole abdomen were observed. Focused assessment with sonography for trauma revealed massive hemoperitoneum. Based on abdominopelvic computed tomography (CT) scans from a previously visited local hospital, she had suffered severe blunt trauma with a grade $\mathrm{V}$ liver laceration, a grade IV right kidney laceration, and suspicious mesenteric root injury (Fig. 1). The injury severity score was 43 . Initial arterial blood gas analysis revealed a $\mathrm{pH}$ of 7.16 , lactate level of $8.1 \mathrm{mmol} / \mathrm{L}$, and hemoglobin level of $13.3 \mathrm{~g} / \mathrm{dL}$, which decreased to $11.9 \mathrm{~g} / \mathrm{dL}$ upon subsequent evaluation. As the patient was confirmed to have hemoperitoneum without any thoracic injuries, REBOA was inserted and threaded into the thoracic aorta to achieve inflow occlusion (Fig. 2). When the balloon was inflated with $16 \mathrm{~mL}$ of saline, the patient's systolic blood pressure rose from $60 \mathrm{mmHg}$ to approximately $90 \mathrm{mmHg}$. The balloon was partially deflated to be $12 \mathrm{~mL}$ and not adjusted afterwards (Fig. 3). She was then intubated and taken to the operating room for crash laparotomy after total 6 units of blood transfusion (Fig. 4).

Upon opening the abdomen, a major liver injury with active bleeding was identified; therefore, rapid perihepatic packing was performed. Inspection of the mesenteric root revealed the presence of a Zone I retroperitoneal hematoma. Instead of exploring the mesenteric root, attention was directed toward the treatment of the shattered right
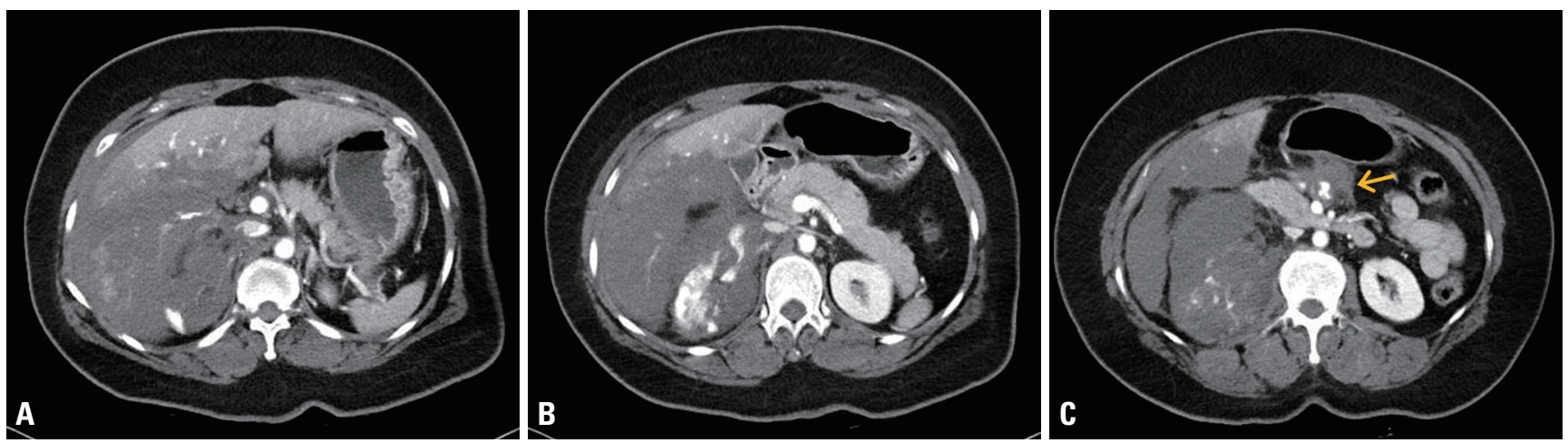

Fig. 1. Preoperative computed tomography of the abdomen. (A) Grade V liver laceration with extravasation. (B) Grade IV right kidney laceration with active bleeding. (C) Hematoma from the mesenteric root injury (arrow). 
kidney. Nephrectomy was initiated using right-sided medial visceral rotation, the Cattell-Braasch maneuver, where the ascending colon and small bowel were shifted aside. The right renal vessels were temporarily clamped following proximal and distal control of the inferior vena cava (IVC). After completion of the Cattell-Braasch maneuver, the renal hilum was ligated and the kidney was extracted. Subsequently, we tried to obtain hemostasis in the bleeding liver using the Pringle maneuver and observed exsanguinating hemorrhage from the retrohepatic IVC. While resecting the liver using the Kelly fracture method, we observed hemoperitoneum in the base of the SMV. Belated dissection of the gastrocolic ligament and exposure of the mesocolon and pancreatic head were performed; however, multiple attempts at primary repair of the SMV failed. The SMV was finally ligated at its proxi-

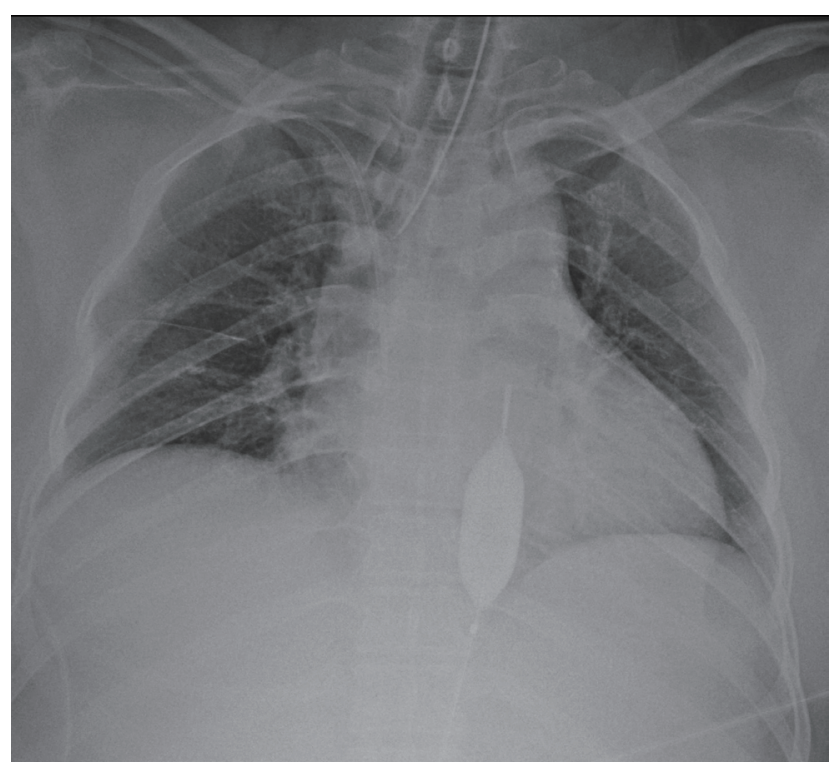

Fig. 2. Position of the resuscitative endovascular balloon occlusion of the aorta (REBOA) in the aortic Zone I. mal and distal portions. However, uncontrollable bleeding was observed in the liver and the patient died 3 hours after temporary abdomen closure with hepatic packing (Supplementary Video 1).

\section{DISCUSSION}

Inflow occlusion is one of the basic principles used to control vascular injury. In a traumatic patient with profound hypovolemic shock, inflow occlusion of the aorta helps in managing noncompressible torso bleeding as well as in improving central hemodynamics [7]. REBOA is the latest technique that can be used as an adjunct to control major abdominal hemorrhage. According to the American Association for the Surgery of Trauma Aortic Occlusion for

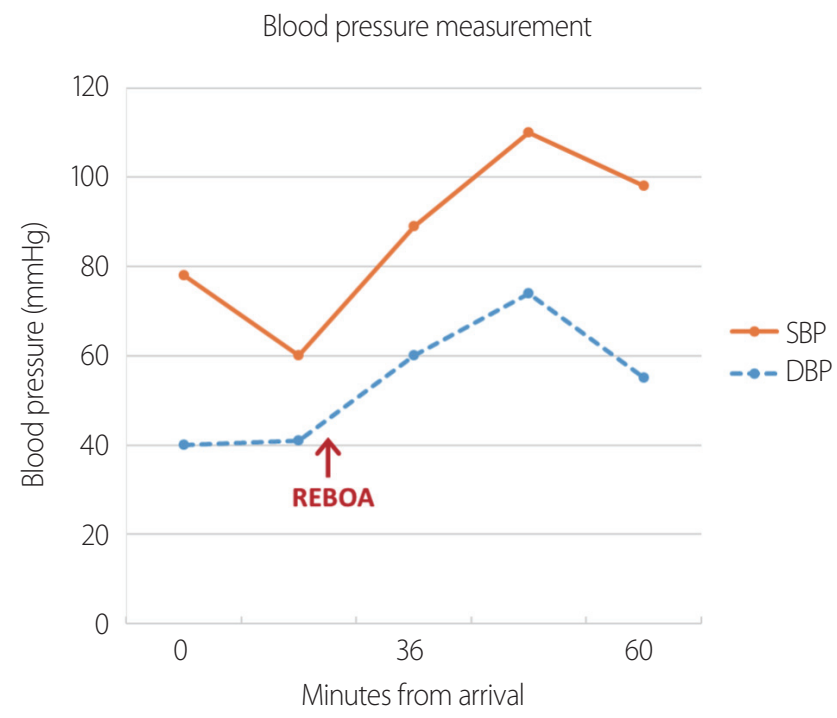

Fig. 3. Blood pressure measurement during preoperative resuscitation. The arrow indicates time of REBOA inflation. REBOA: resuscitative endovascular balloon occlusion of the aorta, SBP: systolic blood pressure, DBP: diastolic blood pressure.

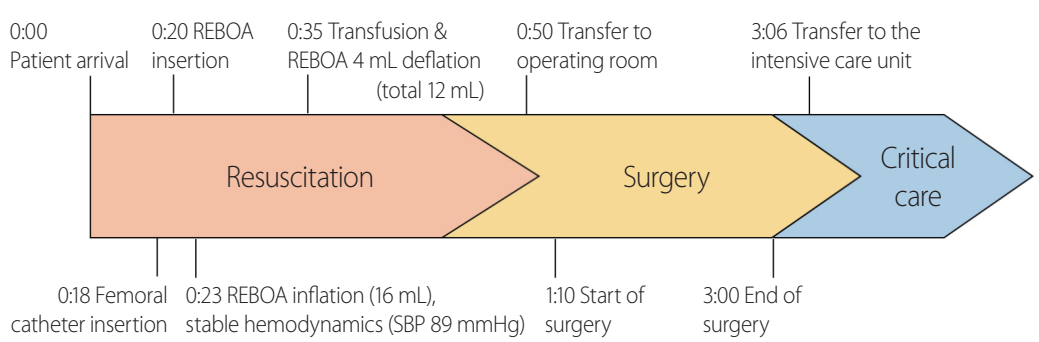

Fig. 4. Time course during REBOA performance (time interval from arrival). REBOA: resuscitative endovascular balloon occlusion of the aorta, SBP: systolic blood pressure. 
Resuscitation in Trauma and Acute Care Surgery registry, compared with resuscitative thoracotomy, REBOA has a higher survival benefit for patients with noncompressible truncal injuries who undergo Zone I REBOA [8]. While resuscitating the patient, we successfully used REBOA as a bridge to DCS following resistant hypotension. Our experience allows for the further application of REBOA for multiple abdominal traumas, including SMV injury, as a bridge to definitive hemostasis.

Determining which damage to repair first among the multiple injuries in DCS is a challenge for every trauma surgeon. In our case, operative intervention of the injured liver and kidney was prioritized rather than inspection of the SMV because bleeding from the solid organs was more easily noticeable. Given the fact that the patient could not survive the exsanguinating hemorrhage from the mesenteric root and liver at the end, a different strategy may have been required during DCS.

Perihepatic packing, which was the first step in our DCS, is the most successful and widely used method for the management of severe liver injury [9]. To enable hemostasis as effective as that by a tampon, compression must be applied from two opposite sides using the surgeon's hands and retractors on solid-surrounding organs. Lap packs should be placed between the liver and diaphragm superiorly, between the liver and rib cage anterolaterally, and between the liver and right kidney or between the liver and transverse colon inferiorly [7]. In our case, inferior hepatic packing could not serve as an

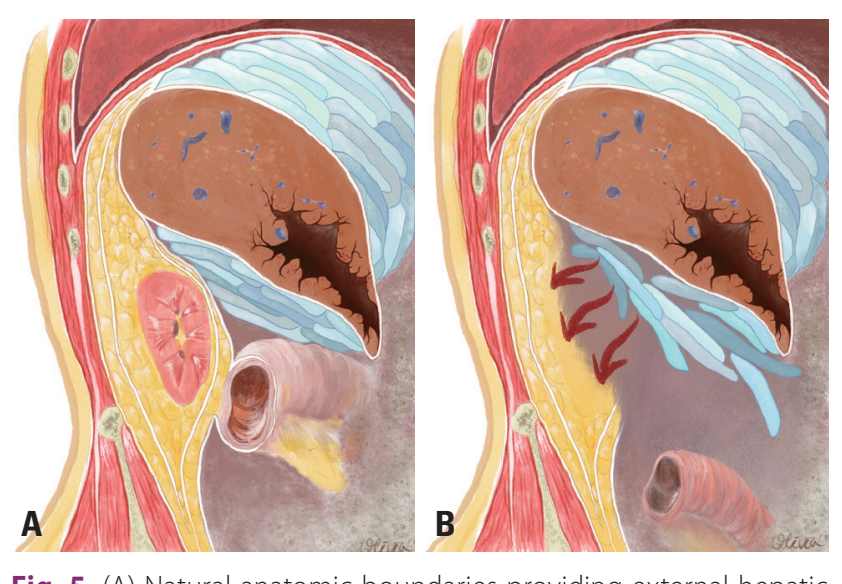

Fig. 5. (A) Natural anatomic boundaries providing external hepatic compression. (B) The inferior tamponade is compromised after right nephrectomy. effective tampon after right nephrectomy because the durable underlay had vanished (Fig. 5). It would have been judicious to approach the crashed kidney and complete nephrectomy after exploring the SMV injury such that hemoperitoneum could have been avoided, which was induced by the loosened inferior hepatic packing.

In a study of 51 patients with traumatic SMV injuries, the patients had an average of 3.5 associated injuries, suggesting that isolated SMV injury is exceedingly rare [6]. The concomitantly injured organs typically include the small bowel, pancreas, IVC, duodenum, stomach, colon, liver, abdominal aorta, and kidney [10]. Although no optimal management of SMV injury has been determined so far, there is a general agreement that DCS is the first choice of treatment [6]. Patients with SMV injuries commonly present with inframesocolic Zone I retroperitoneal hematomas, as diagnosed via CT or as visible in the surgical field. Ligation has become a more acceptable option in the recent history of debates regarding whether repair or ligation should be performed for SMV injuries [11,12]. Reflecting on our surgical management, it was time consuming to attempt SMV repair multiple times rather than to consider early ligation of the vessel. The surgeon should decide on primary repair versus ligation based on the patients' hemodynamic status and associated injuries [6]; SMV repair is not a suitable option in unstable patients. Portal hypertension, venous thrombosis, and bowel ischemia should be monitored because such complications occur as a consequence of splanchnic sequestration of the blood [11].

The Cattell-Braasch maneuver is used to expose the entire inframesocolic retroperitoneal organs, including the IVC, right renal pedicle, right iliac vessels, duodenum, and head of the pancreas. Once the right colon is fully mobilized, the mesenteric root is the only structure supporting the weight of the entire small intestine. When mobile mesentery sustains shearing forces on the mesenteric vessels, it results in unexpected hemorrhage, which is the most serious complication during the procedure [13]. Therefore, care must be taken not to cause iatrogenic traction damage when this maneuver is performed in cases of suspicious SMV injury; otherwise, it could lead to further deterioration of the injury.

Also, lack of hybrid-angio room in our trauma cen- 
ter leaves much to be desired. A hybrid approach by a multi-disciplinary team enables concomitant endovascular intervention and surgery, which saves time in the management of patients with severe multiple traumas and improves their outcomes [14]. The patient might have been saved if she underwent REBOA deployment, DCS and angioembolization of bleeders at the same time in such facility during the golden hour of survival.

In summary, it is important to understand the anatomic mechanism of perihepatic packing. One should set up special plans where there is no natural anatomic boundary to provide pressure against the oozing liver while life-threatening retroperitoneal vascular injuries are addressed and controlled. Definite treatment options for liver injuries, such as liver resection or an atriocaval shunt, should be considered in the very last stage of DCS. SMV injuries require a thorough knowledge of anatomical exposures, and vascular ligation can be a tolerable option for unstable patients. Moreover, the Cattell-Braasch maneuver is an excellent technique for exposing the inframesocolic retroperitoneum; however, it may result in the vulnerability of mesenteric root injury, including the SMV.

To conclude, a systemized surgical strategy in the DCS of multiple abdominal injuries is warranted for patient survival, particularly when there is a concomitant major vessel injury. A more optimal surgical outcome would have been achieved if the order of DCS in our case had been perihepatic packing, exploration of the SMV, nephrectomy, and then damage control of the crashed liver.

\section{SUPPLEMENTARY MATERIALS}

The online-only data supplement is available with this article at https://doi.org/10.20408/jti.2020.0040.

\section{REFERENCES}

1. Zacharias SR, Offner P, Moore EE, Burch J. Damage control surgery. AACN Clin Issues 1999;10:95-142.
2. Schreiber MA. The beginning of the end for damage control surgery. Br J Surg 2012;99(Suppl 1):10-1.

3. Matsushima K, Conti B, Chauhan R, Inaba K, Dutton RP. Novel methods for hemorrhage control: resuscitative endovascular balloon occlusion of the aorta and emergency preservation and resuscitation. Anesthesiol Clin 2019;37:171-82.

4. Accola KD, Feliciano DV, Mattox KL, Burch JM, Beall AC Jr, Jordan GL Jr. Management of injuries to the superior mesenteric artery. J Trauma 1986;26:313-9.

5. Fraga GP, Bansal V, Fortlage D, Coimbra R. A 20-year experience with portal and superior mesenteric venous injuries: has anything changed?. Eur J Vasc Endovasc Surg 2009;37:87-91.

6. Asensio JA, Petrone P, Garcia-Nuñez L, Healy M, Martin M, Kuncir E. Superior mesenteric venous injuries: to ligate or to repair remains the question. J Trauma 2007;62:668-75.

7. Ivatury R. Treatment of liver injuries: an overview. In: Lucas CE, Ledgerwood AM, eds. Operative techniques for severe liver injury. New York:Springer;2015:9-28.

8. Brenner M, Inaba K, Aiolfi A, DuBose J, Fabian T, Bee T, et al. Resuscitative endovascular balloon occlusion of the aorta and resuscitative thoracotomy in select patients with hemorrhagic shock: early results from the American Association for the Surgery of Trauma's Aortic Occlusion in Resuscitation for Trauma and Acute Care Surgery Registry. J Am Coll Surg 2018;226:73040.

9. Moore E, Feliciano D, Mattox K. Liver and biliary tract. In: Bruns B, Kozar R, eds. Trauma 8th ed. New York:McGraw-Hill;2017:551-73.

10. Bourland WA, Kispert JF, Hyde GL, Kazmers A. Trauma to the proximal superior mesenteric artery: a case report and review of the literature. J Vasc Surg 1992;15:669-74.

11. Stone HH, Fabian TC, Turkleson ML. Wounds of the portal venous system. World J Surg 1982;6:335-41.

12. Donahue TK, Strauch GO. Ligation as definitive management of injury to the superior mesenteric vein. J Trauma 1988;28:541-3.

13. Moore E, Feliciano D, Mattox K. Trauma laparotomy: principles and techniques. In: Salotto J, Jurkovich GJ, eds. Trauma. 8th ed. New York:McGraw-Hill;2017:523-38.

14. Kim SH, Song S, Cho HS, Park CY. Hybrid approach for treatment of multiple traumatic injuries of the heart, aorta, and abdominal organs. Korean J Thorac Cardiovasc Surg 2019;52:372-5. 
Yoonjung Heo, et al. Priority in Damage Control Surgery

Supplementary Video 1. Damage control surgery for liver, kidney, and superior mesenteric vein injuries with crash laparotomy. 